\title{
Performance of Web Proxy Caching in Heterogeneous Bandwidth Environments
}

\author{
Anja Feldmann Ramón Cáceres Fred Douglis Gideon Glass Michael Rabinovich \\ AT\&T Labs-Research, Florham Park, NJ, USA
}

\begin{abstract}
Much work on the performance of Web proxy caching has focused on high-level metrics such as hit rates, but has ignored low-level details such as "cookies," aborted connections, and persistent connections between clients and proxies as well as between proxies and servers. These details have a strong impact on performance, particularly in heterogeneous bandwidth environments where network speeds between clients and proxies are significantly different than speeds between proxies and servers.

We evaluate through detailed simulations the latency and bandwidth effects of Web proxy caching in such environments. We drive our simulations with packet traces from two scenarios: clients connected through slow dialup modems to a commercial ISP, and clients on a fast LAN in an industrial research lab. We present three main results. First, caching persistent connections at the proxy can improve latency much more than simply caching Web data. Second, aborted connections can waste more bandwidth than that saved by caching data. Third, cookies can dramatically reduce hit rates by making many documents effectively uncachable.
\end{abstract}

\section{INTRODUCTION}

The continued growth of the World Wide Web (WWW) motivates techniques to improve its performance. One popular technique is proxy caching, in which one or more computers act as a cache of documents for a set of WWW clients. These clients are configured to send HyperText Transport Protocol (HTTP) requests to the proxy. If possible, the proxy serves the requests from its cache. Otherwise, the proxy forwards the request to the content provider, that is, to the server containing the source copy of the requested data.

WWW proxy caching attempts to improve performance in three ways. First, caching attempts to reduce the user-perceived latency associated with obtaining Web documents. Latency can be reduced because the proxy cache is typically closer to the client than the content provider. Second, caching attempts to lower the network traffic from the Web servers. Network load can be lowered because documents that are served from the cache typically traverse less of the network than when they are served by the content provider. Finally, proxy caching can reduce the service demands on content providers since cache hits need not involve the content provider.

Many attempts to model the benefits of proxy caching have looked only at high-level details. For instance, one might consider a stream of HTTP request-response pairs, knowing the URL requested and the size of each response, and compute a byte hit ratio: the fraction of the number of bytes served by the cache divided by the total number of bytes sent to its clients. Hit ratios estimate the reduction in bandwidth requirements between the proxy and the content providers; however, they do not necessarily reflect the latency perceived by the end user, and in some cases they do not even reflect actual bandwidth savings. (The work of Kroeger, et al. [14], is a notable exception in its at- tention to end-user latency. However, it is directed at a different computing environment, a corporate LAN/WAN.)

This paper considers performance implications of factors that cannot be modeled at such high level. In particular, we evaluate the performance effects of sometimes drastically different bandwidth between clients and the proxy and between the proxy and the Internet. Other factors that we consider include aborted transfers, HTTP headers that may affect cachability of resources, and persistent TCP connections.

We have developed a web proxy cache simulator that provides the level of detail necessary to model these factors. It uses workloads from two different environments. One trace was collected in a heterogeneous bandwidth production environment, AT\&T WorldNet. The second trace was collected at an industrial research lab, AT\&T Labs-Research. The simulator considers HTTP operations at the level of individual TCP packets, including the slow-start phase at the beginning of each connection. This simulation includes data in transit, demonstrating the effects of connection aborts. It uses all relevant HTTP request and response headers, so information that affects cachability, such as the presence of cookies, is included. Finally, it uses a combination of measured and parameterizable timing characteristics, allowing us to simulate a user community on a relatively slow modem pool, or a faster local area network.

Our study found that the bandwidth mismatch between the clients and the proxy and the proxy and the Internet can negate any bandwidth savings one might hope to reap from a proxy cache. Without due care, the bandwidth consumption may increase, due to partially transferred data for aborted requests. We also observed that in such heterogeneous networks, the benefits of using persistent connections between clients and the proxy can outweigh the benefits of caching documents. We therefore argue for a dual role of the proxy as a data cache and a connection cache. Relative to a non-caching proxy, an infinite-size data cache reduces average user-observed latency in the lowbandwidth environment by only $8 \%$. Yet, just caching connections alone results in up to $25 \%$ improvement in the average latency. Combined data and connection caching produces up to $28 \%$ latency reduction. In the high-bandwidth environment, data caching improves mean latency by $38 \%$, connection caching improves it by $35 \%$, and the combination of the two improves it by $65 \%$.

Another finding is a surprisingly high fraction of responses with a cookie header. Since cookies are methods of customizing resources on a per-user basis, it is inappropriate to cache 


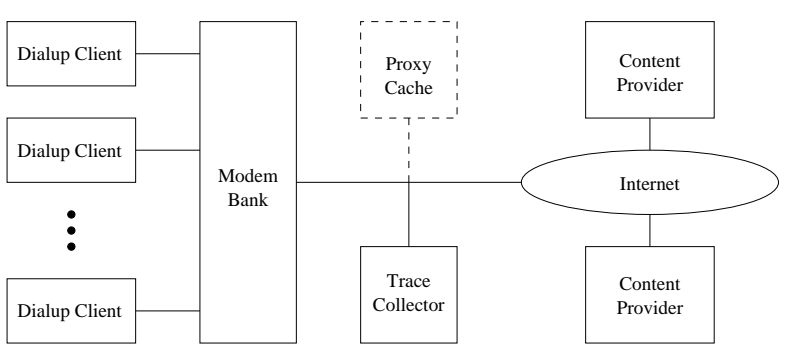

Fig. 1. Trace gathering and proxy caching environment for AT\&T WorldNet.

HTTP $1.0^{1}$ resources that have cookies in them even though they might just be inlined images. In our client trace, we found roughly $30 \%$ of requests had cookies, which dramatically limits the number of requests that can possibly be satisfied from a proxy cache. Moreover, we have evidence that the use of cookies is increasing.

The rest of this paper is organized as follows. Section II describes the tracing environment, and Section III the simulator. Section IV presents our results. Section V discusses related work and Section VI concludes.

\section{TRACING ENVIRONMENT}

Our study is based on two traces, reflecting two common network environments for Internet access. One, referred to as the modem trace, contains accesses by subscribers to a commercial ISP, connected through slow modem lines. The other, called the research trace, contains accesses from a research community connected to the Internet via a high-speed link.

The modem trace was collected from a FDDI ring that connects an AT\&T WorldNet modem bank to the rest of the Internet. In this environment, the proxy caches would reside in the ISP's facilities near such modem pools. Figure 1 depicts this scenario. The modem bank in question contains roughly 450 modems connected to two terminal servers, and is shared by approximately 18,000 dialup users. The servers terminate Pointto-Point Protocol (PPP) connections and route Internet Protocol (IP) traffic between the users and the rest of the Internet.

We collected raw packet traces on a dedicated 500-MHz Alpha workstation attached to the FDDI ring. We ensured that the monitoring was purely passive by configuring the workstation' s FDDI controller so that it could receive but not send packets, and by not assigning an IP address to the FDDI interface. We controlled the workstation by connecting to it over an internal network that does not carry user traffic. The traces were anonymized as soon as they came off the FDDI link, before writing any packet headers to stable storage.

We traced all dialup traffic on the FDDI ring for 12 days in mid-August 1997. During this time, 17,964 different users initiated 154,260 different dialup sessions, with a maximum of 421 simultaneously active sessions. Our tracing infrastructure handled more than 150 million packets a day with less than $0.3 \%$ packet loss. We obtained a second trace of all dialup traffic on the FDDI ring for 6 days in mid-July, 1998.

We processed the raw packet stream on the fly to obtain our

\footnotetext{
${ }^{1}$ In HTTP 1.1, caching and cookies are decoupled, and a server is responsible for explicitly disabling caching when appropriate.
}

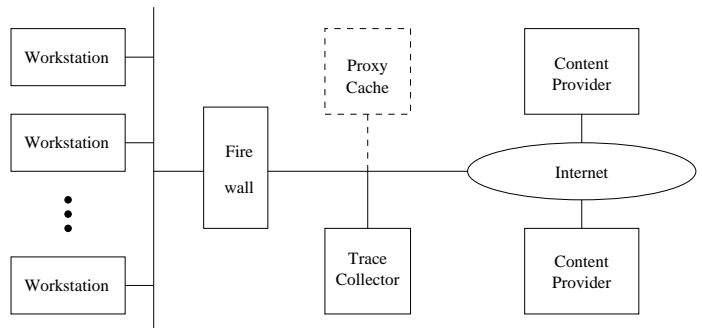

Fig. 2. Trace gathering environment for AT\&T Labs-Research.

final trace. The resulting trace contains all relevant information and is much more compact than the raw packet data. The trace records the following information for TCP conversations in which one port number is the default HTTP port (80):

- TCP events: Timestamps, sequence numbers, and acknowledgments for all packets with SYN, FIN, or RST bits set.

- HTTP events: Timestamps for HTTP requests from clients and HTTP responses from servers, and timestamps for the first and last data packets in each direction.

- HTTP headers: Complete HTTP headers for both requests and responses.

- Byte counts: Count of bytes sent in either direction for HTTP header and body, if present.

This information is sufficient to determine how much time is taken by various components of the observed HTTP conversations. These traces are significantly more detailed than traces that other researchers have made available (e.g., [11], [14]). For example, publicly available traces lack timestamps for TCP events such as connection creation (SYN packets).

For the research trace, we recorded accesses from the AT\&T Labs-Research community. The tracing environment for collecting the research trace is shown in Figure 2. The trace was gathered from an Ethernet segment adjacent to the serial line that connects AT\&T Labs-Research to the rest of the Internet. We used the same trace collection software as in the modem case. At the time of the trace collection this serial line was a T1 link (1.5 Mbit/s). The raw packet traces were collected on a dedicated 133-MHz Intel Pentium PC running Linux. Again we isolated the tracing from the traced network.

We traced the high-speed clients for 11 days in mid-February 1997. During this time, more than 3,000 client machines accessed more than 23,000 external Web servers.

\section{SimUlATOR}

Our Web proxy simulator, named PROXIM, simulates a proxy cache using the HTTP trace described in Section II as input. PROXIM can be used to simulate the three different scenarios shown in Figure 3: (a) using a proxy, (b) without a proxy, where the bandwidth to the clients is the bottleneck, and (c) without a proxy, where the bandwidth on the network connecting the clients to the Internet is the bottleneck.

To assess the performance impact of proxy caching in a given environment, we compare the with-proxy to the no-proxy simulation results. In addition, the no-proxy simulation results are used to validate the simulator against the original measured numbers. The next subsections describe various aspects 


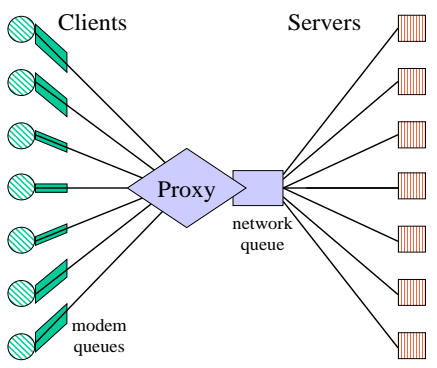

(a) Using a proxy.

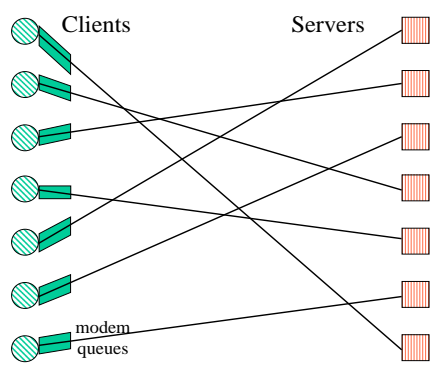

(b) No-proxy; client bandwidth is the bottleneck.

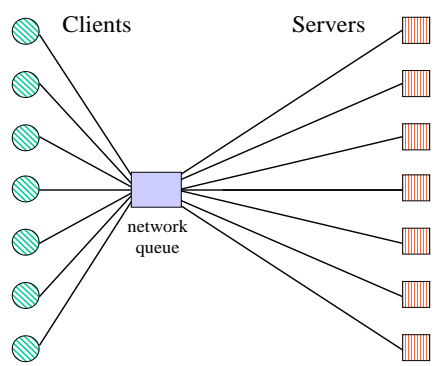

(c) No-proxy; bandwidth to the Internet is the bottleneck.

Fig. 3. Proxy caching environment.

of PROXIM's functionality while the last subsection discusses the validation.

Simulated Cache: PROXIM simulates a document cache managed by LRU replacement. However, in all of our experiments we configured the cache size to be sufficiently large to avoid having to evict any documents. This gives potentially optimistic results for the proxy, but the storage consumed by all cachable documents in our trace is on the order of $40 \mathrm{~GB}$, not an unreasonable amount of disk space. To account for the overhead introduced by the proxy, a configurable cache miss time overhead and a configurable cache hit time overhead are added to the request service time.

Network Connections: As mentioned in the introduction, it is important to handle the interactions between HTTP and TCP, particularly persistent HTTP [9], [16], [19]. In PROXIM, each simulated client maintains zero or more open connections to the proxy. An idle connection is one in which no HTTP request is currently active. When a persistent HTTP request is generated and an idle connection exists for the client in question, an idle connection is chosen to service the request. By default, requests that were marked non-persistent in the original trace (due to client or server header fields) are treated as non-persistent by PROXIM and result in a new connection. If a client generates a request and all of its extant proxy connections are active servicing other requests - or if the client has no extant connectionsa new TCP connection is created by the client to service the present request. Client-to-proxy connections that are idle for more than a default of 3 minutes are closed by the proxy.

PROXIM handles proxy to content-provider connections similarly to the way it handles proxy to client connections. However, since proxy administrators have no influence over arbitrary content providers, we time out persistent server connections after a somewhat arbitrary default of 30 seconds of idle time.

PROXIM assumes that a client requests a Web page at the time that the original client sent out the SYN packet (TCP connection request). Therefore, if the client can reuse a persistent connection, then the proxy learns about an HTTP request at the time at which we recorded the SYN packet. If the client is forced to open a new connection to the proxy, the proxy sees the request at one client-to-proxy round-trip time (RTT) after the original SYN packet was observed.

Document Transfer: PROXIM simulates the transfer of doc- uments to clients over a connection by scheduling individual packets to arrive at the client, the proxy, or the server. The scheduling of packets takes into account TCP slow-start at the start of a connection, but does not consider packet losses and the additional slow-start periods those would entail. By default, the proxy and servers send 1500-byte packets (the most common packet size besides 40-byte ACK packets).

Packets destined for the clients are enqueued in a per-client queue for scenarios (a) and (b) and in a network queue for scenario (c) of Figure 3. Packets destined for the proxy are enqueued in a network queue. Each of these queues drains at a configurable rate. The default for the modem trace is a serverto-proxy rate of $45 \mathrm{Mbps}$ while the default rate for client queues is $21 \mathrm{Kbps}$. The first was chosen since a T3 (45 Mbps) constitutes a good ISP connectivity to the Internet and the latter was chosen since most modems connection speeds at this modem pool are in the order of $24 \mathrm{Kbps}$ to $28 \mathrm{Kbps}$ and we observed that they can sustain a throughput around $21 \mathrm{Kbps}$. The default for the research trace is a server-to-proxy rate of $1.5 \mathrm{Mbps}$ and a network queue of 1.5 Mbps. These values were chosen since a T1 (1.5 Mbps) constitutes typical connectivity to the Internet for a corporate environment.

This scheduling of packets requires a RTT estimate for the network link on which packets are scheduled. We use a constant RTT estimate for each type of connection. For clientto-proxy connections in the modem trace, the RTT is the modem delay (default $250 \mathrm{~ms}$ ). For proxy-to-server connections the RTT is derived from either the difference between the SYN and SYN-ACK timestamps or the REQUEST and RESPONSE timestamps. (We assume that the proxy is located at the location of the packet sniffer, where the trace was collected.) The estimate for the client-to-server connection is done in a similar manner except that we add the modem delay of $250 \mathrm{~ms}$ in the case of the modem trace. For the research trace we assume a default round-trip delay of $2.5 \mathrm{~ms}$ between the clients and the proxy.

Latency Calculations: PROXIM simulates the overall latency to retrieve a document by breaking the latency overhead into connection setup time, HTTP request-response time, and document transfer time. Connection setup costs are avoided when a persistent connection is reused. In the no-proxy case, we use the SYN timestamps in the trace to determine the con- 
nection setup time. In the with-proxy case, we use the relevant subset of the SYN packet timestamps. Similarly, requestresponse latency is calculated using the appropriate portion of the HTTP request/reply latencies in the trace: the full latency is used if the proxy has to connect to the content provider; otherwise the request/response latency is assumed to be the configured client/proxy RTT. We modeled the time clients take to retrieve data from the proxy by using the queues mentioned above, which drain at modem bandwidth. We break documents into packets and observe the time difference between the start of the first downloaded packet and the dequeuing of the last packet.

Simulator Validation: To validate the simulator, we compare our results for the no-proxy simulations where either the client bandwidth is the bottleneck (modem environment) or where the bandwidth to the Internet is the bottleneck (research lab environment) and compared simulated latencies against the original latencies from the trace. Figure 4 shows the probability density of latency distribution in both cases. The simulation curve matches the general shape of the measured curve.

In particular, the median latencies for the data transfers in the original trace in the modem environment is 0.44 seconds vs. 0.42 seconds for the no proxy simulation. The median total latency for the no-proxy simulation is 2.4 seconds vs. 1.8 seconds for the original trace. This difference is due to the overestimation of message RTT in the simulator, which is calculated as the minimum of SYN/SYN-ACK and HTTP request/response delays, both of which require extra server processing. On the other hand, the mean latency of the original trace is larger than the mean latency of the no-proxy simulation since the no-proxy simulation does not account for network congestion or content server overload, which might limit the data deposit rate into the modem queues. Note that this simulation is assuming that the client bandwidth is the bottleneck.

For the research environment, most of the clients are connected via a 10Mbit/s Ethernet. Therefore we follow the scenario of Figure 3 (c). In this case we choose the bandwidth of the network queue to be $1.5 \mathrm{Mbps}$ (T1 speed) and assume that the network queue is drained at $6 \mathrm{Mbps}$ (a reasonable assumption given a $10 \mathrm{Mbps}$ Ethernet). The median latencies for the original trace in the research environment is 0.42 seconds vs. 0.54 seconds for the no-proxy simulation. Again the mean latency of the original trace exceeds the mean latency of the no-proxy simulation. Overall, both simulations reasonably represent the original trace.

\section{Performance EfFects of the Proxy}

This section describes the insights gained from considering the impact that low-level details can have on the performance of Web proxies.

\section{A. Hit Ratio:}

Most performance studies of proxies to date have concentrated on hit ratios. We contend this is only a secondary measure, useful insofar as it affects the performance metrics that users and network administrators ultimately care about, such as bandwidth savings and latency reduction.
Still, even when looking at just hit ratio, considering lowlevel details provides some interesting results. Unlike existing studies, our trace captures all HTTP headers of requests and responses, so we were able to emulate the full behavior of proxies with respect to cachability of resources. There are a variety of reasons for a document to be uncachable:

- Dynamically generated content, usually identified by the presence of "cgi-bin" or "?" in the URL.

- Explicit cache control through the use of Cache-Control and Expires header fields [9].

- A cookie is present: by using a Set-Cookie HTTP response header line, the content-provider has requested that the client send the content-provider a "cookie" with each subsequent request. The cookie is an arbitrary sequence of bytes, which is sent by a client by means of a Cookie header line. Cookies are often used for authorization and/or personalization of Web pages. Although the Cookie and Set-Cookie header fields are not (yet) officially part of HTTP and thus have no inherent caching-related requirements, current proxy caching software should not cache the results of HTTP 1.0 requests with Cookie header lines. By default, proxy software (e.g., Squid [22]) generally does not cache results of requests with Cookie header lines, but (as described below) some vendors of commercial caches weaken this constraint in some cases such as images.

- An authorization field is present in the request: this would require proxies to cache content on a per-client basis, or unauthorized users might see content meant for another user.

The following table lists the number of HTTP requests that were uncachable for various reasons. The counts are nonexclusive, but the last line of the table counts the union of the individual causes.

\begin{tabular}{l||r|r||r|r}
\hline \multicolumn{1}{l||}{ Reason } & \multicolumn{2}{c||}{ Modem } & \multicolumn{2}{c}{ Research } \\
& \multicolumn{1}{c||}{ Count } & \multicolumn{1}{c}{$\%$} & Count & \multicolumn{1}{c}{$\%$} \\
\hline \hline Cookie present & $3,236,869$ & 30.20 & 196,071 & 18.83 \\
URL had '?' & $1,055,470$ & 9.85 & 77,692 & 7.46 \\
Client Cache-Control & 800,574 & 7.47 & 149,720 & 14.38 \\
URL had 'cgi-bin' & 580,451 & 5.42 & 48,753 & 4.68 \\
Neither GET nor HEAD & 216,073 & 2.02 & 19,336 & 1.86 \\
Authorization present & 180,728 & 1.69 & 11,505 & 1.10 \\
Server Cache-Control & 177,404 & 1.65 & 11,412 & 1.10 \\
\hline \hline Uncachable docs & $4,615,661$ & 43.06 & 391,064 & 37.55 \\
\hline
\end{tabular}

Surprisingly, the ISP trace showed that over $30 \%$ of all requests had a cookie, which had a dramatic effect on the hit ratio. If the proxy ignored cookies in the ISP, the hit ratio would have been $54.5 \%$, similar to hit rates observed in previous studies [11], [14]. By taking cookies into account, we observe a hit ratio of just $35.2 \%$, even when the cache size is large enough to avoid any purges. The byte hit ratio similarly decreases from $40.9 \%$ to $30.4 \%$. In fact, we have preliminary evidence that the use of cookies is increasing. In a six-day trace we collected in June 1998, the fraction of requests with cookies grew to $34.5 \%$.

For the somewhat older research trace, the impact of cookies is not quite as dramatic. The hit rate decreases from $33.9 \%$ to $27.0 \%$ if the proxy considers responses with cookies uncachable; the byte hit rate decreases from $28.1 \%$ to $23.3 \%$. The fact that the hit rate is much lower for the research trace than 


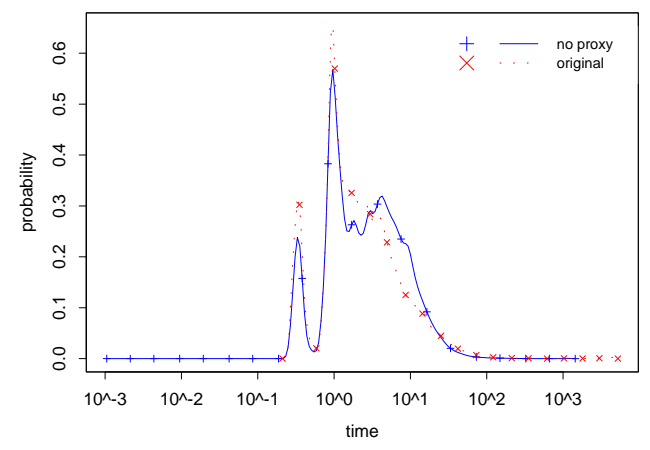

(a) Modem environment

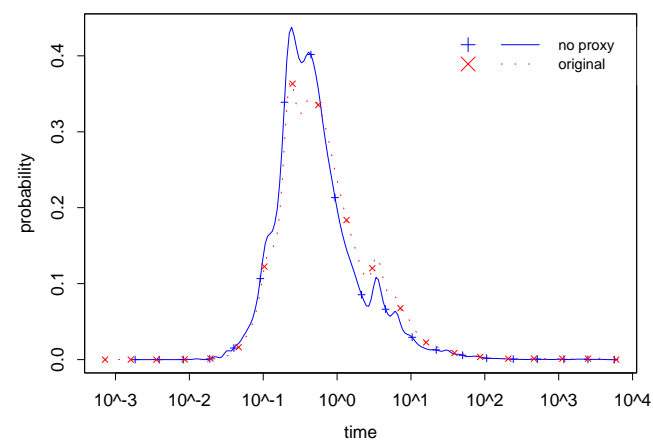

(b) Research environment

Fig. 4. Simulation validation: total latency.

for the modem trace is likely to be correlated with the lower request rate of the research trace and the difference in browsing behavior for researchers and consumers.

The significant increase of hit ratios produced by caching documents with cookies supports the importance of techniques aimed at enabling caching of such of documents. These techniques include approaches based on delta encoding [1], [13], [18] and client-side HTML macro-preprocessing [6]. The extent of the benefits of these techniques would depend on the amount of document customization based on the request cookies.

Another technique used by some proxy vendors is to ignore a cookie if the request appears to be for an image (identified by a suffix in the URL) [5]. This technique is based on the heuristic that images are rarely personalized and therefore the presence of a cookie in this case is simply the result of sloppy Web site design.

While HTTP 1.1 explicitly allows caching cookied documents unless prohibited by the content server, HTTP 1.0 does not officially support a cookie header. In practice, caching these documents can result in unpredictable behavior.

\section{$B$. Bandwidth Consumption:}

An often-cited benefit of proxy caching is reduced network traffic on the Internet. Surprisingly, by carefully modeling the system behavior when requests are aborted, we found that the proxy can actually increase the traffic from content providers to the ISP. Without a proxy, when a user aborts a request, by hitting the "stop" button, the transfer of the document is interrupted and no longer consumes network bandwidth beyond what is already in transit. On the other hand, due to the bandwidth mismatch between the connections from client to proxy and from proxy to the Internet, a proxy that retrieves documents at full speed may have downloaded much or all of the document by the time it learns of the abort. Thus the bandwidth consumption of aborted requests can be higher when the proxy is present.

The following table indicates the frequency of interrupted transfers for the ISP trace. While some browsers will abort a connection using the RST flags within TCP, others will just close the connection. Therefore, aborts are hard to infer purely based upon the state of the connection. Instead we infer which doc- uments are aborted by relating HTTP response Content-length header fields to the amount of data that was actually received by the clients (excluding header data). This is possible for responses with valid Content-length (i.e. a non-zero value). In the table below the first line corresponds to completed HTTP transfers; the second line in the table indicates incomplete HTTP transfers (about $11 \%$ of requests); the third line is an exceptional case-the server sent more data than it claimed as the document's Content-length. Presumably these responses are due to buggy server software.

\begin{tabular}{l|r|r|r|r}
\hline Case & Count & $\%$ & $\begin{array}{r}\text { Content-Length } \\
\text { Bytes }\end{array}$ & $\begin{array}{r}\text { Response } \\
\text { Bytes }\end{array}$ \\
\hline \hline CL = RDL & $5,487,519$ & 88.79 & $33731 \mathrm{MB}$ & $33731 \mathrm{MB}$ \\
$\mathrm{CL}>$ RDL & 676,243 & 10.94 & $44483 \mathrm{MB}$ & $8210 \mathrm{MB}$ \\
$\mathrm{CL}<\mathrm{RDL}$ & 16,706 & 0.27 & $131 \mathrm{MB}$ & $151 \mathrm{MB}$ \\
\hline
\end{tabular}

The exact effect of aborts on the overall bandwidth demands depends on the handling of aborted requests by the proxy. If the proxy continues the download, subsequent requests to the document will hit in the cache, improving performance. On the other hand, this may further increase the traffic from the content providers to the proxy.

In our simulations, the total number of bytes received by the clients from the content providers without the proxy was 49.8 GB. At one extreme, if the proxy always continued to download upon aborts, the total number of bytes from the content providers to the proxy would be $58.7 \mathrm{~GB}$ (118\% of the original number of bytes transferred from the content providers). This means that, with the proxy, one could end up consuming $18 \%$ more bandwidth to the Internet.

On the other extreme, the proxy could abort the download immediately after receiving a client's abort. In this case, the the amount of wasted data transmitted depends on the proxy-toserver bandwidth and the rate at which data are requested.

The following table compares bandwidth consumption of the network with and without the proxy, for various values of the proxy-to-server bandwidth. We are initially assuming documents are retrieved at the full bandwidth of the proxy-to-server connection. 


\begin{tabular}{l|r|r}
\hline Proxy-server & Data transfered & Savings \\
\hline \hline 45Mbps & $53.7 \mathrm{~GB}$ & $-8 \%$ \\
$1.5 \mathrm{Mbps}$ & $50.7 \mathrm{~GB}$ & $-2 \%$ \\
$0.5 \mathrm{Mbps}$ & $41.5 \mathrm{~GB}$ & $15 \%$ \\
\hline
\end{tabular}

As seen from this table, for the network queue bandwidth of $45 \mathrm{Mbps}$, aborting downloads would reduce wasted data transmissions by only $5.0 \mathrm{~GB}$, still an increase of $8 \%$ over the without-proxy case. A $1.5 \mathrm{Mbps}$ Internet connection would correspond to a $2 \%$ increase in overall traffic. However, if the bandwidth of the network queue were just $0.5 \mathrm{Mbps}^{2}$, savings from caching data would finally offset any additional transfers after the abort, amounting to $15 \%$ savings overall. These bandwidth savings (if any) from the proxy are nowhere near those suggested by the byte hit ratio.

Using the AT\&T Labs trace from clients connected to the Internet via a T1 link, we confirmed that the bandwidth mismatch between the modems and the Internet contributes significantly to the wasted bandwidth consumption from aborts. When a wellconnected client aborts a transfer, it will have received a significant fraction of the data received by the proxy. Handling of aborts is important even in the case of well-connected clients, however, since if the proxy continues to download the document even after an abort, it will see minimal savings. If the proxy aborts immediately it will see 14\% savings in bandwidth.

We should note that some cache implementations include flow control over the proxy-to-server connection (e.g., Network Appliance [5] and Infolibria [12]). In these implementations, a slow client causes the TCP buffer for the proxy-to-server connection to fill up and stall the data inflow. To evaluate this feature, we simulated a proxy that would throttle its inflow from the content server to be no more than $64 \mathrm{~KB}$ ahead of the outflow to the client. This turned an $8 \%$ increase in bandwidth consumption for the $45 \mathrm{Mbps}$ case into a $13 \%$ savings.

Cache flow control remains a controversial issue, with some vendors (e.g., Cisco [10]) trying to completely decouple the rate of receiving data from servers and sending it to clients. Our data indicate that cache flow control is important in achieving bandwidth savings in a heterogeneous network.

\section{Latency Reduction: Caching Connections vs. Caching Data}

Multiple components within a typical HTTP request contribute to the latency of that request. Depending on the bandwidth environment, adding a proxy cache can influence the latency of any of these components and therefore change the latency experienced by the user in several ways.

Latency Components: Most document downloads proceed as follows: (1) the client establishes a TCP connection to the server; (2) the client sends the HTTP request for a document; (3) the server sends the HTTP response for the document; (4) the server sends the data for the document; (5) the server/client closes the TCP connection. With persistent connections [16] it is possible to skip steps (1) and (5). In the modem and research

\footnotetext{
${ }^{2}$ Note that the lower-bandwidth network queues are appropriate models given that a lot of web sites are accessed over a network path that includes at least one $\mathrm{T} 1$ or lower speed connection.
}

traces respectively, $18 \%$ and $12 \%$ of the HTTP requests are sent over persistent connections.

Figure 5 illustrates the breakdown of latency components for our original traces without a proxy in place. ${ }^{3}$ The latencies do not include the modem delays. Note that connection creation contributes a significant amount of time to the end-to-end latency. The heavy-tailed nature of the document transfer (the mean time is much larger than the median) [4] implies that there are numerous documents ( $47 \%$ for the modem trace and $26 \%$ for the research trace) for which the connection setup time is at least $50 \%$ of the total download time.

Due to the high cost of TCP connection set-up, potentially significant benefits could be obtained by maintaining persistent connections between clients and servers. However, content providers can maintain only a limited number of such connections. This suggests that a proxy may also serve in the nontraditional role of a connection cache: it would maintain persistent connections with content providers and re-use them for obtaining documents for multiple clients. The connection cache requires only one (or a small number of) persistent connection(s) to a given content provider, regardless of the number of clients connected to the proxy. Similarly, each client needs to maintain only a small number of persistent connections to the proxy regardless of the number of servers it visits. Moreover, if for some reason a connection between the proxy and client or between the proxy and content provider is closed, significant performance benefits could still be obtained due to the connection with the other side.

Modem Trace-Latency Analysis: For the modem trace Figure 6 shows the effects of both serving data from the cache (data caching), and using persistent connections (referred to as "connection caching"), on the total latency. One would expect that those requests that are served from the cache will be serviced faster. However, we see from Figure 6(a) that data caching reduces the latencies experienced by the user by only a minimal margin. The two curves fall more or less on top of each other, except for the peaks for small latency values in both the withproxy and no-proxy simulations. The peak in the no-proxy simulation reflects the use of persistent connection in the trace itself. The peak in the with-proxy simulation, at about $500 \mathrm{~ms}$, reflects the minimum amount of time that it takes to establish a connection and exchange the HTTP request/response information and some data given a round-trip time of $250 \mathrm{~ms}$. In total, the improvement relative to the no-proxy simulation in the mean and median latencies is $3 \%$ and $4 \%$ respectively.

This result is substantially more pessimistic than the upper bound of $26 \%$ latency reduction reported by Kroeger et al. [14]. The limited improvement is due to the heterogeneous bandwidth environment, which implies a high latency of the connection setup (which had a mean of $1.3 \mathrm{~s}$ in the trace) and a modem bandwidth limitation on the clients (this study assumed $28.8 \mathrm{Kbps}$ modems). We may be treating the pure data cache unfairly by

\footnotetext{
${ }^{3}$ The graphs plot the density of the logarithm of the latencies. Coupled with a logarithmic scale on the $x$-axis, plotting the density of the logarithm of the data facilitates direct comparisons between different parts of the graphs based on the area under the curve.
} 


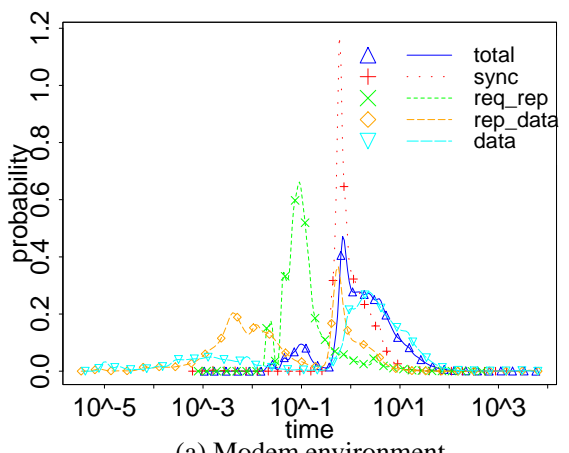

(a) Modem environment

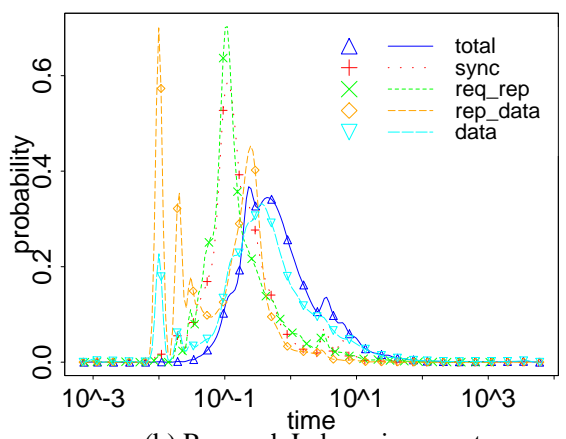

(b) Research Lab environment

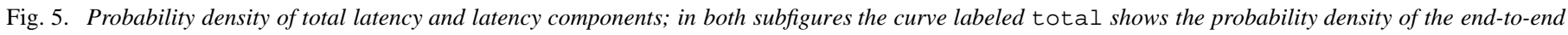
request latency; the curve labeled sync shows the latency to establish the TCP connection from the client to the content-provider; the curve labeled req_rep shows the time between sending the HTTP request to receiving the HTTP response; the curve labeled rep_dat a shows the time between receiving the HTTP response and the first part of the document; finally the curve labeled dat a shows the transfer time for requests in which a document was returned.

not allowing the use of persistent connections for those requests that used persistent connection in the original trace. Compared to a non-caching proxy, data caching improves the mean performance by $8 \%$.

Figure 6(b) verifies our intuition that if the proxy is used as a connection cache, the total mean latency improvements grow substantially. In this heterogeneous bandwidth environment the probability shifts from the larger latencies for the no-proxy case to substantially shorter latencies for the with-proxy case. The mean and median latencies improve by $21 \%$ and $40 \%$ respectively. Indeed, now the first peak of both curves reflects HTTP requests over persistent connections, while the second peak in the no-proxy curve reflects the time it takes to establish the TCP connections.

The with-proxy curve of Figure 6(c), which shows the total latency for a Web proxy that is acting both as a connection cache and a data cache, differs only slightly from the with-proxy curve of Figure 6(b). Indeed, the mean and median latencies improves by $24 \%$ and $48 \%$ respectively. On first thought it may be surprising that the improvement in the median is larger than the individual components would suggest. Data caching can improve the performance of the connection cache by shortening transfers and therefore making a connection available for reuse by a different transfer at an earlier time. This explains why the performance improvements of data caching plus connection caching can be more than additive.

The overhead of setting up TCP connections can be assessed by looking at the latency between when a client issues a request for a document and the time when the Web proxy has acted on the HTTP request (either by serving it out of the cache or by forwarding it to the server). The benefit of data caching can be better understood from the latency between the sending of the HTTP request and receiving the HTTP response message. Unfortunately, in the heterogeneous bandwidth environment the latency between the client and the proxy is in the same order of magnitude as the latency between the client and the server, and is not a large proportion of the overall latency. Therefore the benefit of data caching is limited. Also, the mean latency of the TCP connection setup is larger than the mean latency of the HTTP handshake, which somewhat reduces the importance of data caching. (Additional details are available in a technical report [8].)

Modem Trace-Caching Connections vs. Caching Data: With the understanding of which latencies are influenced by data/connection caching, it is clear that the benefit from connection caching will dominate the total latency improvements for the heterogeneous bandwidth environment. Various connection cache scenarios to consider are: all connections are persistent, only connections from the Web proxy to the Web servers are persistent, only connections from the Web client to the Web proxy are persistent, or none of the connections are persistent. Figure 7 summarizes the performance improvements of various different ways of combining persistent connections with data caching, including "cheating" on documents with cookies by declaring them cachable.

Given that a proxy introduces overheads, it is not too surprising that using no persistent connections and no data caching will imply an increased latency. Persistent connections between the clients and the Web proxy are not as effective as between the Web proxy and the Web server since they only save the round-trip time between the Web proxy and the Web server. The performance improvements of introducing persistent TCP connections only between the Web server and the Web proxy are larger than introducing a data cache for documents without cookies and without persistent connections. Adding persistent client connections to caching improves the latency results by more than a factor of 2. Adding all persistent connections improves the latency results over only data-caching by more than a factor of 7.5.

Caching documents improves the median latencies significantly more than the mean latencies since small documents are much more likely to be served from the cache. This means that each individual transfer that uses a persistent connection will occupy the connection for a smaller amount of time. This in turns means that it is more likely that another document transfer can reuse the connection. Note that enabling caching of documents with cookies will improve the mean latency only from $24 \%$ to $26 \%$ in the scenario with all persistent connections. A more significant improvement from $3 \%$ to $8 \%$ can be achieved in the 


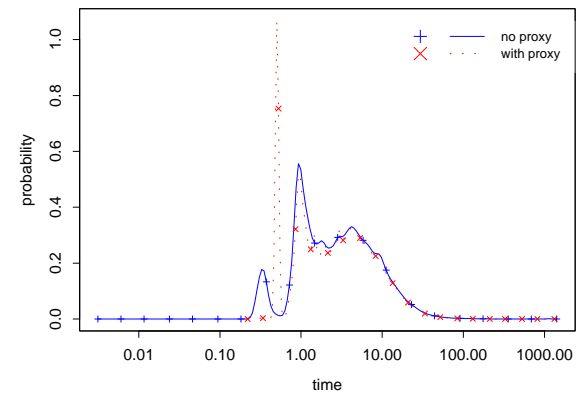

(a) Data cache only

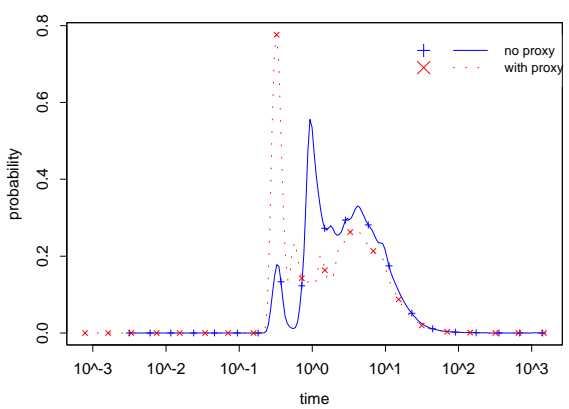

(b) Connection cache only

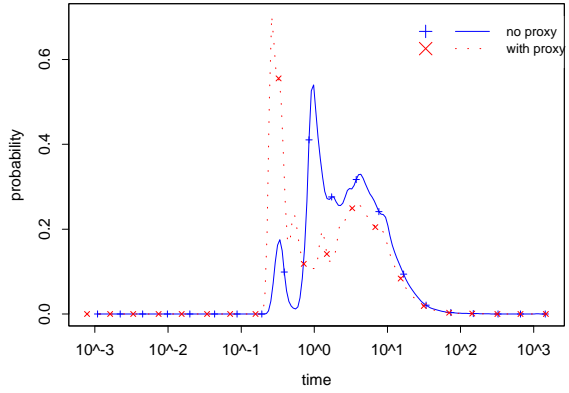

(c) Data and connection cache

Fig. 6. Total latency comparison for AT\&T WorldNet modem trace

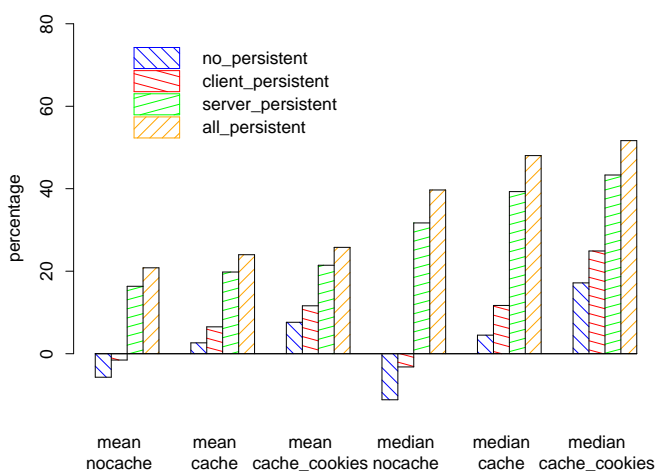

Fig. 7. Latency improvement results for the AT\&T WorldNet modem trace relative to the no-proxy case.

scenario with no persistent connections.

Research Trace: Latency Analysis: Our simulation results for the AT\&T WorldNet trace show that connection caching is highly beneficial in heterogeneous bandwidth environments. To understand the difference between this environment and an environment with a set of well connected clients, Figure 8 shows the total latency comparison for the AT\&T Labs-Research trace for the same scenarios as Figure 6.

In this environment we see a mean latency improvement of $7 \%$ for the pure data cache proxy (see Figure 8(a)). This is a factor of 2 improvement over the modem environment. At first glance, pure connection caching (see Figure 8(b)) yields only a small 2\% mean latency improvement compared to the no-proxy results. (The median improves by 20\%.) Given that the noproxy simulation is allowed to use persistent connections for those document transfers that used persistent connections in the original trace, this comparison may not be appropriate. Comparing the results to the no-caching/no-persistent-connection case we observe a mean latency improvement of $39 \%$ for the research trace, but only an improvement of $8 \%$ for the modem trace. When adding connection caching to data caching (see Figure $8(\mathrm{c})$ ) the mean and median latencies improve by $47.5 \%$ and $40.4 \%$ respectively. This is in the same order of magnitude as the improvements from no persistent connections to all persistent connections.

Comparing the individual latency components in the two environments gives insights into how and why the results for the research trace differ from the modem trace. Because of the small

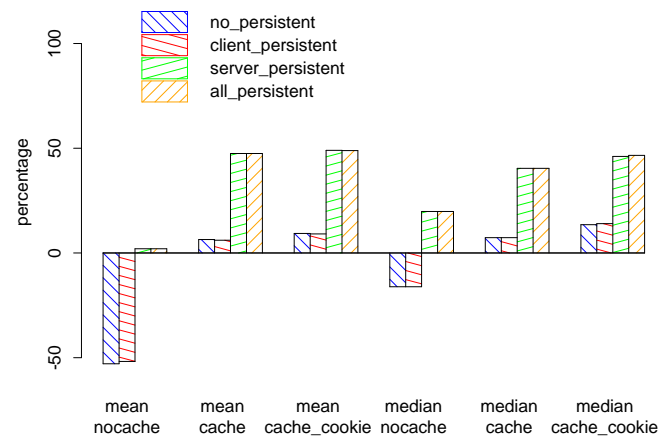

Fig. 9. Latency improvement results for the AT\&T Labs-Research trace relative to the no-proxy case.

round-trip time, the HTTP request to HTTP response latency for cached documents is very small for the research environment and the connection speed to the clients is not a bottleneck. This is reflected very positively in the document transfer times. Small documents can be retrieved much faster with a proxy than without a proxy.

Research Trace: Caching Connections vs. Caching Data: Figure 9 reflects results for the same scenarios as Figure 7 of combining various forms of connection caching with data caching for the AT\&T Labs-Research trace. The results for no cache and no persistent connections show a substantial slowdown. The slowdown is partially caused by the fact that these simulations have to establish new connections for document transfers that in the original trace and in the no-proxy simulation use persistent connections. Moreover, in a fast network, the proxy introduces possible penalties due to interactions between two TCP windows.

Because document caching reduces the document transfer latencies substantially in a low latency environment we see that connection caching and data caching are highly complimentary. In sum they improve the performance by more than $40 \%$. Given that the client to proxy latencies are so small using persistent connections between the Web proxy and the Web server will be sufficient to gain most of the benefit while the benefit of using persistent connection between the Web clients and the Web proxy is almost nonexistent.

Data caching results in substantially better latency improvements (a factor of 2) in the high bandwidth environment, even 


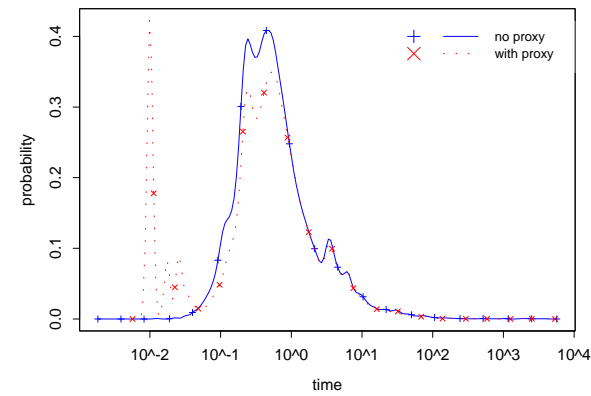

(a) Data cache only

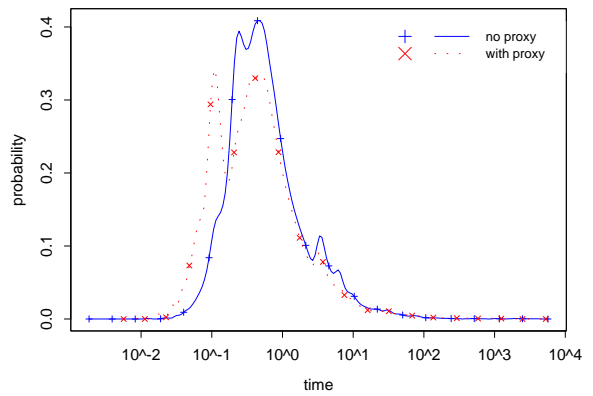

(b) Connection cache only

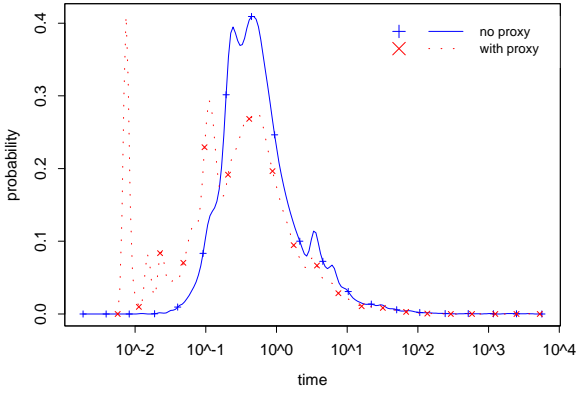

(c) Data and connection cache

Fig. 8. Total latency comparison for AT\&T Labs-Research trace

with a lower hit rate, than in the heterogeneous bandwidth environment. Overall combining data caching with connection caching has a mean and median improvement of $47.5 \%$ and $40.5 \%$ respectively.

\section{Connection Cache Size}

Connection caching can produce substantial performance benefits. Therefore, we need to identify the caching overheads, such as the cache size requirements. PROXIM maintains a list of currently active connections. If a connection between a client and a Web proxy is idle for more than 3 minutes it is purged from the cache. Connections to servers are cached for 30 seconds after last use. The next two tables summarize the maximum number of simultaneous connections maintained by a data caching proxy for different connection caching scenarios. The first column of the tables specifies what connections are cached by the proxy. The next two columns contain the maximum connection numbers, which in combination indicate the capacity requirements for the proxy. The last two columns represent the percent of client requests that resulted in opening a new connection, respectively, between a client and the proxy, and the proxy and a server. Note that in the case of proxy-to-server connections, the proxy may avoid opening a new connection by serving the data from the cache or by reusing another connection.

Number of connections in modem environment

\begin{tabular}{l|r|r|r|r}
\hline $\begin{array}{l}\text { Connections } \\
\text { cached }\end{array}$ & $\begin{array}{r}\text { max \# TCP } \\
\text { conn to client }\end{array}$ & $\begin{array}{r}\text { max \# TCP } \\
\text { conn to server }\end{array}$ & $\begin{array}{r}\% \text { conn } \\
\text { to client }\end{array}$ & $\begin{array}{r}\% \text { conn } \\
\text { to server }\end{array}$ \\
\hline \hline all & 646 & 240 & 18.4 & 20.6 \\
proxy/server & 203 & 243 & 100.0 & 20.8 \\
proxy/client & 646 & 75 & 18.4 & 77.8 \\
none & 204 & 73 & 100.0 & 77.87 \\
\hline
\end{tabular}

Number of connections in research environment

\begin{tabular}{l|r|r|r|r}
\hline $\begin{array}{l}\text { Connections } \\
\text { cached }\end{array}$ & $\begin{array}{r}\max \text { \# TCP } \\
\text { conn to client }\end{array}$ & $\begin{array}{r}\max \text { \# TCP } \\
\text { conn to server }\end{array}$ & $\begin{array}{r}\% \text { conn } \\
\text { to client }\end{array}$ & $\begin{array}{r}\% \text { conn } \\
\text { to server }\end{array}$ \\
\hline \hline all & 216 & 198 & 10.1 & 25.2 \\
proxy/server & 179 & 198 & 100.0 & 25.2 \\
proxy/client & 216 & 183 & 10.1 & 84.0 \\
none & 179 & 183 & 100.0 & 84.0 \\
\hline
\end{tabular}

In the modem environment caching connections may increase the maximum number of connections by more than a factor of 3. If this should exceed the facilities of the Web proxy, just turning off persistent client connections will reduce the overhead to only a factor of 1.6 while preserving most of the latency benefits. To judge the impact of persistent connection caching on the capacity requirements of the Web server, we compare the total number of simultaneous connections without caching or persistent connections (112) to the number of connections from the Web proxy to the Web server (240). This is an increase of slightly more than a factor of 2 .

The costs of using connection caching in the research environment is very small with a factor of less than 1.3. The requirements for the Web servers are actually reduced by a factor of 2 in this case. Overall, the connection cache hit rates are very promising given that the percentage of new connection establishments is reduced by at least a factor of 4 for both environments.

When using persistent connections on average a persistent connection between the client and the proxy will be used for 5.4 requests while a persistent connection between the proxy and the server will be used for 3.8 requests. On average a persistent connection between a client and a proxy will be used by requests that go to two different Web servers and the persistent connection between a proxy and a server will be used by requests from 1.2 different clients. Note that these are means! Some of the connections will be used for requests from many more clients and to many more servers. This means that there is substantial diversity in how persistent connections are used that cannot be achieved without a proxy.

The cost of connection caching can be reduced further by identifying better policies for managing the connection cache. This includes answering questions as when to tear down a connection, which connection to tear down when the proxy runs out of connections, how many simultaneous connections should be maintained to a given content provider or client, when to wait for a persistent connection instead of opening a new connection, and so on [3].

\section{RELATED WORK}

From the standpoint of analyzing modem users, the work that is most similar to ours is that of Gribble and Brewer [11]. They collected a trace of HTTP accesses over a modem pool at the University of California, Berkeley, over a period of 45 days and including over 8,000 clients. They used this trace to evaluate several metrics, including the effectiveness of proxy caching and 
the load placed on servers. We agree with some of their conclusions, particularly the need for efficient support of a large number of TCP connections. We disagree in our enthusiasm with respect to the amount of locality in the clients' reference stream; we do not agree that a "large amount" of locality is present. Clearly there is enough locality to make caching modestly useful, but with cache misses outnumbering cache hits, the miss case is the common case and needs to be handled well. We believe the difference in our conclusions is due to our assumption that the existence of cookies make a resource uncachable, while they do not mention cookies at all.

Other research on reducing client latency complements our work, particularly to the extent that modem bandwidth is the apparent bottleneck. Kroeger, et al., analyzed the effect of proxycaching on latency rather than just bandwidth [14]. Using the widely-used Digital trace, they found that proxy caching could reduce latency by only up to $26 \%$, even though the cache hit ratio was roughly $50 \%$. However, their particular study focussed on corporate LANs and WANs, so they did not consider the heterogeneous bandwidth environment we have evaluated here. Other approaches to latency reduction include prefetching [21], [14], data compression [18], [19], and delta-encoding [13], [1], [18].

Duska, et al. [7], used a variety of proxy cache logs to evaluate hit rates, locality, sharing, and cache coherence. They found that proxy cache hit rates varied from $24 \%$ to $45 \%$, with higher request rates resulting in higher hit rates. They focused on resource hit rates and byte hit rates, but not latency effects.

Persistent connections were first proposed by Padmanabhan and Mogul [20] and simulated by Mogul [17]. They are now part of the HTTP 1.1 proposed standard [9]. Most previous analysis of persistent connections have concerned persistent connections between clients and servers [15], [19] rather than connection caching by a proxy, or focussed on slow-start effects [23] rather than connection establishment latency.

\section{SUMMARY}

In this paper, we presented results of a detailed simulation study of Web proxy caching in heterogeneous bandwidth environments. We made three main observations. First, latency reductions from caching persistent connections can be much greater than those from caching data. The two types of caching are complementary and should be used together. Second, the bandwidth saved by caching can be more than offset by the bandwidth wasted by aborted connections. It is important to manage aborted connections well. Third, hit ratios can be severely reduced by the growing percentage of documents containing cookies. More study is needed into effective ways to cache documents with cookies. An important overall lesson was that low-level details have a significant impact on all aspects of system performance: hit ratios, bandwidth utilization, and userperceived latency. These details should be considered in future studies.

\section{ACKNOWLEDGMENTS}

We would like to thank Albert Greenberg and John Friedmann for their help with the data collection effort. Thanks also to Peter Danzig and Jennifer Rexford for helpful discussions.

A summary of preliminary results of this study was reported at the ACM SIGMETRICS Workshop on Internet Server Performance [2].

\section{REFERENCES}

[1] Gaurav Banga, Fred Douglis, and Michael Rabinovich. Optimistic deltas for WWW latency reduction. In Proceedings of 1997 USENIX Technical Conference, pages 289-303, Anaheim, CA, January 1997.

[2] Ramón Cáceres, Fred Douglis, Anja Feldmann, Gideon Glass, and Michael Rabinovich. Web proxy caching: The devil is in the details. In ACM SIGMETRICS Workshop on Internet Server Performance, June 1998. Available at http://www.cs.wisc.edu/cao/WISP98/final-versions/anja.ps.

[3] Edith Cohen, Haim Kaplan, and Jeffrey Oldham. Policies for managing TCP connections under persistent HTTP. Unpublished, November 1998.

[4] Mark E. Crovella and Azer Bestavros. Self-similarity in World Wide Web traffic: Evidence and possible causes. In Proceedings of 1996 ACM SIGMETRICS Conference, 1996.

[5] Peter Danzig, 1998. Personal communication.

[6] Fred Douglis, Antonio Haro, and Michael Rabinovich. HPP: HTML macro-preprocessing to support dynamic document caching. In Proceedings of the Symposium on Internet Technologies and Systems, pages 83-94. USENIX, December 1997.

[7] Bradley M. Duska, David Marwood, and Michael J. Feeley. The measured access characteristics of World-Wide-Web client proxy caches. In Proceedings of the Symposium on Internet Technologies and Systems, pages 23-35. USENIX, December 1997.

[8] Anja Feldmann, Ramón Cáceres, Fred Douglis, Gideon Glass, and Michael Rabinovich. Performance of web proxy caching in heterogeneous bandwidth environments. Technical report, AT\&T, January 1999.

[9] R. Fielding, J. Gettys, J. Mogul, H. Frystyk, T. Berners-Lee, et al. RFC 2068: Hypertext transfer protocol - HTTP/1.1, January 1997.

[10] Samuel D. Gendler, 1998. Personal communication.

[11] Steven D. Gribble and Eric A. Brewer. System design issues for internet middleware services: Deductions from a large client trace. In Proceedings of the Symposium on Internet Technologies and Systems, pages 207-218. USENIX, December 1997.

[12] Abdelsalam Heddaya, 1998. Personal communication.

[13] Barron C. Housel and David B. Lindquist. WebExpress: A system for optimizing Web browsing in a wireless environment. In Proceedings of the Second Annual International Conference on Mobile Computing and Networking, pages 108-116, Rye, New York, November 1996. ACM.

[14] Thomas M. Kroeger, Darrell D. E. Long, and Jeffrey C. Mogul. Exploring the bounds of web latency reduction from caching and prefetching. In Proceedings of the Symposium on Internet Technologies and Systems, pages 13-22. USENIX, December 1997.

[15] Stephen Manley and Margo Seltzer. Web facts and fantasy. In Proceedings of the Symposium on Internet Technologies and Systems, pages 125-133. USENIX, December 1997.

[16] Jeffrey Mogul. The case for persistent-connection HTTP. In Proceedings of ACM SIGCOMM'95 Conference, pages 299-313, October 1995.

[17] Jeffrey Mogul. The case for persistent-connection HTTP. Computer Communication Review, 25(4):299-313, October 1995.

[18] Jeffrey Mogul, Fred Douglis, Anja Feldmann, and Balachander Krishnamurthy. Potential benefits of delta-encoding and data compression for HTTP. In Proceedings of ACM SIGCOMM'97 Conference, pages 181194, September 1997.

[19] Henrik Frystyk Nielsen, James Gettys, Anselm Baird-Smith, Eric Prud'hommeaux, Hakon Wium Lie, and Chris Lilley. Network performance effects of HTTP/1.1, CSS1, and PNG. In Proceedings of ACM SIGCOMM'97 Conference, pages 155-166, September 1997.

[20] Venkata N. Padmanabhan and Jeffrey C. Mogul. Improving http latency. Computer Networks and ISDN Systems, 28(1-2):25-35, December 1995.

[21] Venkata N. Padmanabhan and Jeffrey C. Mogul. Using predictive prefetching to improve World Wide Web latency. Computer Communication Review, 26(3):22-36, 1996.

[22] Squid internet object cache. http://squid.nlanr.net/, 1997.

[23] Joe Touch, John Heidemann, and Katia Obraczka. Analysis of http performance. USC/Information Sciences Institute. Available as http://www.isi.edu/lsam/publications/http-perf/. 